The Astrophysical Journal, 604:484-494, 2004 April 1

C) 2004. The American Astronomical Society. All rights reserved. Printed in U.S.A.

\title{
EARLY REIONIZATION BY MINIQUASARS
}

\author{
P. Madau, ${ }^{1,2}$ M. J. Rees,${ }^{3}$ M. Volonteri, ${ }^{1}$ F. HaARdt ${ }^{4}$ and S. P. $\mathrm{OH}^{5}$ \\ Received 2003 October 9; accepted 2003 December 9
}

\begin{abstract}
Motivated by the recent detection by the Wilkinson Microwave Anisotropy Probe of a large optical depth to Thomson scattering, implying a very early reionization epoch, we assess a scenario where the universe was reionized by "miniquasars" powered by intermediate-mass black holes (IMBHs), the remnants of the first generation of massive stars. Pregalactic IMBHs form within minihalos above the cosmological Jeans mass collapsing at $z>20$, get incorporated through mergers into larger and larger systems, sink to the center as a result of dynamical friction, and accrete cold material. The merger history of dark halos and associated IMBHs is followed by Monte Carlo realizations of the merger hierarchy in a $\Lambda$ CDM cosmology. Our model is based on the assumptions that quasar activity is driven by major mergers and nuclear IMBHs accrete at the Eddington rate a fraction of the gas in the merger remnant. The long dynamical frictional timescales leave many IMBHs "wandering" in galaxy halos after a minor merger. While seed IMBHs that are as rare as the $3.5 \sigma$ peaks of the primordial density field evolve largely in isolation, a significant number of BH binary systems will form if IMBHs populate the more numerous $3 \sigma$ peaks instead. In the case of rapid binary coalescence a fraction of IMBHs will be displaced from galaxy centers and ejected into the intergalactic medium (IGM) by the "gravitational rocket" effect, rather than accrete and shine as miniquasars. We show that, under a number of plausible assumptions for the amount of gas accreted onto IMBHs and their emission spectrum, miniquasars powered by IMBHs, and not their stellar progenitors, may be responsible for cosmological reionization at $z \sim 15$. Reionization by miniquasars with a hard spectrum may be more "economical" than stellar reionization, as soft X-rays escape more easily from the dense sites of star formation and travel farther than EUV radiation. Energetic photons will permeate the universe more uniformly, make the low-density diffuse IGM warm and weakly ionized prior to the epoch of reionization breakthrough, set an entropy floor, and reduce gas clumping. Future $21 \mathrm{~cm}$ observations may detect a preheated, weakly ionized IGM in emission against the cosmic microwave background.
\end{abstract}

Subject headings: black hole physics — cosmology: theory — galaxies: evolution — intergalactic medium — quasars: general

\section{INTRODUCTION}

In popular cold dark matter (CDM) hierarchical cosmogonies, the very first generation of metal-free ("Population III") stars is expected to form in DM "minihalos" of total mass $M_{h} \gtrsim 5 \times 10^{5} M_{\odot}$ (e.g., Fuller \& Couchman 2000; Yoshida et al. 2003), condensing as a result of $\mathrm{H}_{2}$ cooling from the high- $\sigma$ peaks of the primordial density field at redshift $z=20-30$. Recent numerical simulations of the collapse and fragmentation of primordial molecular clouds suggest that the first stars were predominantly very massive, $m_{*} \gtrsim 100 M_{\odot}$, a scale linked to $\mathrm{H}_{2}$ chemistry and cooling (Bromm, Coppi, \& Larson 2002; Abel, Bryan, \& Norman 2002). Zero-metallicity very massive stars have spectra similar to that of a $\sim 10^{5} \mathrm{~K}$ blackbody (Tumlinson \& Shull 2000) and emit about 20 times more Lyman continuum photons per stellar baryon than a standard stellar population (Schaerer 2002; Bromm, Kudritzki, \& Loeb 2001). A "top-heavy" primordial initial mass function

\footnotetext{
${ }^{1}$ Department of Astronomy and Astrophysics, University of California, 477 Clark Kerr Hall, Santa Cruz, CA 95064.

2 Carnegie Observatories, Carnegie Institution of Washington, 813 Santa Barbara Street, Pasadena, CA 91101.

${ }^{3}$ Institute of Astronomy, University of Cambridge, Madingley Road, Cambridge CB3 0HA, UK

${ }^{4}$ Dipartimento di Scienze, Università dell'Insubria/Sede di Como, Italy.

5 Department of Physics, University of California, Santa Barbara, CA 93106
}

(IMF), quite different from the present-day Galactic case, may have then played a crucial role in determining the ionization, thermal, and chemical enrichment history of the intergalactic medium (IGM) at early times.

The IGM is known to be highly ionized at least out to redshift $\sim 5.5$. While the excess $H_{\text {I }}$ absorption measured in the spectra of $z \sim 6$ quasars in the Sloan Digital Sky Survey (SDSS) has been interpreted as the signature of the trailing edge of the cosmic reionization epoch (Djorgovski et al. 2001; Fan et al. 2002; White et al. 2003), the recent detection by the Wilkinson Microwave Anisotropy Probe (WMAP) satellite of a large optical depth to Thomson scattering, $\tau_{e}=0.17 \pm 0.04$ $(68 \%)$, suggests that the universe was reionized at much higher redshift, $z_{\text {rei }}=17 \pm 3$ (assuming instantaneous hydrogen reionization; see Kogut et al. 2003; Spergel et al. 2003). This is an indication of significant star formation activity at very early times. In the wake of $W M A P$ remarkable results, several theoretical studies of stellar reionization scenarios have pointed out that an early generation of massive, metal-free stars with a top-heavy IMF may be necessary to produce a Thomson optical depth as high as the central value determined by WMAP (Cen 2003b; Ciardi, Ferrara, \& White 2003; Haiman \& Holder 2003; Sokasian et al. 2003; Wyithe \& Loeb 2003; Somerville \& Livio 2003).

Since, at zero metallicity, mass loss through radiatively driven stellar winds or nuclear-powered stellar pulsations is expected to be negligible (Kudritzki 2002; Baraffe, Heger, \& 
Woosley 2001), Population III stars will likely die losing only a small fraction of their mass. Nonrotating very massive stars in the mass window $140 M_{\odot} \lesssim m_{*} \lesssim 260 M_{\odot}$ will disappear as pair-instability supernovae (Bond, Arnett, \& Carr 1984), leaving no compact remnants and polluting the universe with the first heavy elements (e.g., Schneider et al. 2002; Oh et al. 2001b; Wasserburg \& Qian 2000). Stars with $40 M_{\odot}<m_{*}<$ $140 M_{\odot}$ and $m_{*}>260 M_{\odot}$ are predicted instead to collapse to black holes (BHs) with masses exceeding half of the initial stellar mass (Heger \& Woosley 2002). Barring any finetuning of the IMF of Population III stars, intermediatemass black holes (IMBHs), with masses above the 4-18 $M_{\odot}$ range of known "stellar-mass" BHs (e.g., McClintock \& Remillard 2003), may then be the inevitable end product of the first episodes of pregalactic star formation. Since they form in high- $\sigma$ density fluctuations, "relic" IMBHs and their descendants will tend to cluster in the bulges of present-day galaxies as their host halos aggregate into more massive systems (Madau \& Rees 2001, hereafter Paper I; Islam, Taylor, \& Silk 2003). The first IMBHs may also have seeded the hierarchical assembly of the supermassive variety (SMBHs) observed at the center of luminous galaxies (Volonteri, Haardt, \& Madau 2003a, hereafter Paper II; Volonteri, Madau, \& Haardt 2003b, hereafter Paper III).

Population III IMBHs accreting gas from the surrounding medium will shine as "miniquasars" at $z \sim 15$ and generate a soft X-ray background that may catalyze the formation of $\mathrm{H}_{2}$ molecules in dense regions and counteract the destruction by UV Lyman-Werner radiation (Cen 2003a; Glover \& Brand 2003; Haiman, Abel, \& Rees 2000). The net effect would be an increase in the cooling rate and star formation efficiency of minihalos (but see Machacek, Bryan, \& Abel 2003, who find the positive feedback effect of X-rays to be quite mild). In this paper we point out that miniquasars represent an additional source of Lyman continuum photons that must be taken into account in models of early reionization by Population III objects. Thin-disk accretion onto a Schwarzschild BH releases about $100 \mathrm{MeV}$ baryon $^{-1}$. If "seed" IMBHs were able to (say) double their initial mass via gas accretion, and just a few percent of the radiated energy were emitted above and close to the hydrogen Lyman edge, then miniquasars would be more efficient at photoionizing the universe than their metal-free stellar progenitors.

While the basic idea is simply stated, it is not easy to quantify and model in detail. A minihalo at $z \sim 20$ will typically undergo several major mergers in a Hubble time. Pregalactic IMBHs will follow the merger history of their hosts and may be able to accrete efficiently only in the inner densest regions of the merger remnant. Because of the long dynamical frictional timescales, many IMBHs will not sink to the center and will be left "wandering" in galaxy halos after a minor merger. If seed holes are numerous enough, binary systems may form in significant numbers. In this case, rather than accrete and shine as miniquasars, a fraction of IMBHs may be displaced from galaxy centers and ejected into the IGM by the "gravitational rocket" effect (Redmount \& Rees 1989) or by triple $\mathrm{BH}$ interactions (Paper II). Miniquasars are expected to be copious sources of soft X-ray photons, which will permeate the IGM more uniformly than possible with extreme ultraviolet (EUV; $\geq 13.6 \mathrm{eV}$ ) radiation $(\mathrm{Oh} 2000,2001)$ and make it warm and weakly ionized prior to the epoch of reionization breakthrough (Venkatesan, Giroux, \& Shull 2001).

In this paper we make a first attempt at treating the growth and impact on the very early IGM of Population III IMBHs in the context of hierarchical structure formation theories, incorporating some of the essential astrophysics into a scenario for the reheating and reionization of the universe by miniquasars. The plan is as follows. In $\S 2$ we outline a model in which seed holes populate the rare $3.5 \sigma$ peaks of the primordial density field. We show that if cold material can be accreted efficiently onto IMBHs hosted in minihalos, then miniquasars may be responsible for cosmological reionization at $z \sim 15$. In $\S 3$ we present results for a case in which seed holes are more numerous but gas accretion occurs less efficiently and address the dynamics of IMBH binary systems in shallow potential wells. In $\S 4$ we discuss the implications of an early reionization and reheating epoch by hard radiation from miniquasars. Finally, we summarize our results in $\S 5$. Unless otherwise stated, all results shown below refer to the currently favored $\Lambda$ CDM world model with $\Omega_{M}=0.3$, $\Omega_{\Lambda}=0.7, h=0.7, \Omega_{b}=0.045, \sigma_{8}=0.93$, and $n=1$.

\section{BUILDUP OF IMBHS IN MERGING MINIHALOS}

A scenario for the birth, growth, and evolution of IMBHs that traces their formation in minihalos with virial temperatures $T_{\text {vir }}<10^{4} \mathrm{~K}$ back to very high redshifts was put forward in Papers I and II. Seed IMBHs are placed far up in the dark halo merger tree, get incorporated into larger and larger halos, sink to the center as a result of dynamical friction, and accrete a fraction of the gas in the merger remnant. The merger history of DM halos and associated IMBHs is followed through Monte Carlo realizations (based on the extended Press-Schechter formalism) of the merger hierarchy from early times to the present.

Here we improve on several aspects and adapt some prescriptions in our model to the conditions expected during the epoch of first light. Pregalactic seed IMBHs form within the mass ranges $20 M_{\odot}<m_{\mathrm{BH}}<70 M_{\odot}$ and $130 M_{\odot}<m_{\mathrm{BH}}<$ $200 M_{\odot}$, as remnants of the first generation of massive metalfree stars that do not disappear as pair-instability supernovae. For simplicity, within these intervals the differential BH mass function is assumed to be flat; i.e., the IMF-averaged BH mass is $115 M_{\odot}$. They form in isolation within minihalos above the cosmological Jeans mass collapsing at $z=24$ from rare greater than $\nu \sigma$ peaks of the primordial density field. As our fiducial model we take $\nu=3.5$, corresponding in the assumed $\Lambda \mathrm{CDM}$ cosmology to minihalos of mass $M_{\text {seed }}=1.3 \times 10^{6} M_{\odot}$. As halos more massive than the $\nu \sigma$ peaks contain a fraction $\operatorname{erfc}(\nu / \sqrt{2}) \quad(=0.00047$ for $\nu=3.5)$ of the mass of the universe, the mass density parameter of our " $3.5 \sigma$ " pregalactic holes is

$$
\Omega_{\mathrm{BH}}=\frac{0.00047 \Omega_{M}\left\langle m_{\mathrm{BH}}\right\rangle}{M_{\text {seed }}}=10^{-7.9} .
$$

This is $0.6 \%$ of the mass density of the supermassive variety found in the nuclei of most nearby galaxies, $\Omega_{\mathrm{SMBH}}=(2.1 \pm$ $0.3) \times 10^{-6}$ (Yu \& Tremaine 2002). We also study a case in which seed holes are more numerous and populate all peaks above $3 \sigma$. Then $\operatorname{erfc}(3 / \sqrt{2})=0.0027, M_{\text {seed }}=1.2 \times 10^{5} M_{\odot}$, and the initial mass density of " $3 \sigma$ " IMBHs is $\Omega_{\mathrm{BH}}=10^{-6.1}$, less than $40 \%$ of the mass density of SMBHs today. We do not consider lighter, more common halos than those originating from $3 \sigma$ fluctuations, as they would fall below the minimum mass threshold for baryonic condensation via molecular hydrogen cooling, $M_{\mathrm{TH}}=1.25 \times 10^{5} M_{\odot}$ according to the simulations of Machacek, Bryan, \& Abel (2001). Seed holes that were much rarer than the $3.5 \sigma$ density peaks would have little 
impact on the reionization of the IGM at $z \gtrsim 15$. In addition, the assumed "bias" assures that almost all halos above $10^{11} M_{\odot}$ actually host a $\mathrm{BH}$ at later epochs. For simplicity, we start our calculations by placing all seed IMBHs in their host halos at $z=24$, the highest redshift we are able to follow the merger hierarchy to because of computational costs. More realistically, IMBHs are expected to form continuously from Population III massive stars over a range of redshifts.

Each halo is treated as a singular isothermal sphere (SIS) with density $\rho \propto r^{-2}$, truncated at the virial radius. During the merger of two halos, the "satellite" progenitor with initial mass $M_{s}$, as well as its IMBH if it hosts one, spirals in toward the center of the more massive preexisting system (with mass $M_{h}>M_{s}$ ) on the dynamical friction (against the DM background) timescale. This depends on the orbital parameters of the satellite (van den Bosch et al. 1999; Ghigna et al. 1998) and on tidal mass loss/evaporation. $N$-body simulations show that massive satellites with mass ratios $P \equiv M_{s} / M_{h}>0.1$ ("major" mergers) sink rapidly without significant mass loss, while the lightest satellites $(P<0.01)$ are almost unaffected by dynamical friction and do not suffer orbital decay (Taffoni et al. 2003). In our calculations we adopt the expression for the dynamical friction timescale of a mass-losing satellite in a main halo suggested by Colpi, Mayer, \& Governato (1999).

In our fiducial model $(\nu=3.5)$, the density of seed holes is small enough that the merging of two minihalos both hosting a $\mathrm{BH}$ is a rare event, and IMBHs evolve largely in isolation. This is shown in Figure 1, where the number of halos containing two or more primordial IMBHs, normalized to the total number of $\mathrm{BH}$ hosts, is plotted as a function of redshift. The ratio, as expected, increases with time and tends to saturate at lower redshifts. For illustrative purposes, the realizations in this figure have been run neglecting any dynamical interaction between BHs along cosmic history. At the very high redshifts of interest here this is a good assumption for $\nu=3.5$ as the fraction of multiple systems is typically less than $10 \%$. If seed holes populate the $\nu=3$ peaks instead, the fraction of multiple $\mathrm{BH}$ hosts can exceed $50 \%$ and bound binaries form in significant numbers. We discuss the dynamics of IMBH binaries in $\S 3$.

\subsection{Accretion onto IMBHs}

Hydrodynamic simulations of major mergers have shown that a significant fraction of the gas in interacting galaxies falls to the center of the merged system (Mihos \& Hernquist 1996): the cold gas may be eventually driven into the very inner regions, fueling an accretion episode and the growth of nuclear BHs (Kauffmann \& Haehnelt 2000; Cavaliere \& Vittorini 2000). Since the local SMBH mass density is consistent with the integrated luminosity density of quasars (Yu \& Tremaine 2002; Fabian 2003), the fraction of cold gas ending up in the hole must depend on the properties of the host halo in such a way as to ultimately lead to the observed correlation between stellar velocity dispersion and SMBH mass (Ferrarese \& Merritt 2000; Gebhardt et al. 2000). In Paper II it was assumed that in each major merger the hole in the most massive halo accretes at the Eddington rate a gas mass that scales with the fifth power of the circular velocity of the host halo: the normalization was fixed a posteriori in order to reproduce the observed local $m_{\mathrm{BH}^{-}} \sigma_{*}$ relation (Ferrarese 2002). Our simple model was shown to explain remarkably well the observed luminosity function of optically selected quasars in the redshift range $1<z<5$. Accretion was inhibited onto all $\mathrm{BHs}$

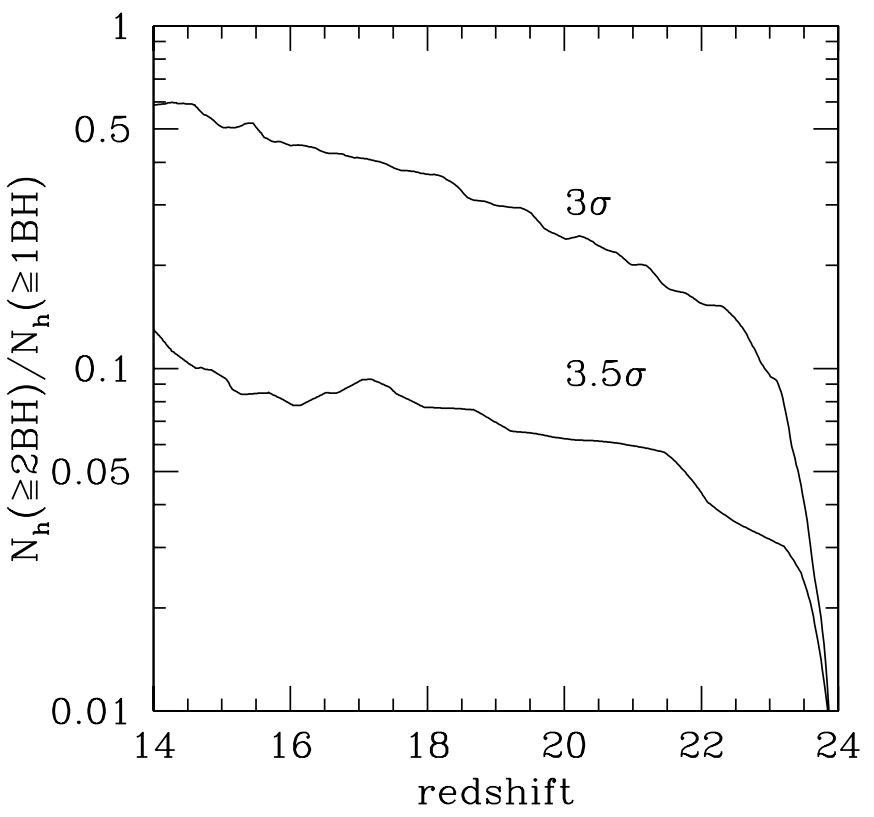

FIG. 1.-Fraction of BH host halos that contain two or more seed IMBHs as a function of redshift, averaged over 50 "trees." Two curves are shown, one assuming that BHs form in isolation within minihalos collapsing at $z=24$ from $3.5 \sigma$ peaks, the other from $3 \sigma$ peaks instead. For illustrative purposes, dynamical interactions between BHs have been switched off along cosmic history.

hosted in halos with virial temperature $T_{\text {vir }} \lesssim 10^{4} \mathrm{~K}$, as below this temperature atomic cooling is not effective in allowing the gas to sink to the center.

Prior to the epoch of reionization, however, cold material may be efficiently accreted onto IMBHs hosted in minihalos. As mentioned in $\S 1$, gas condensation in the first baryonic objects is possible through the formation of $\mathrm{H}_{2}$ molecules, which cool efficiently via rotovibrational transitions even at virial temperatures of a few hundred kelvins. In the absence of a UV photodissociating flux and of ionizing X-ray radiation, three-dimensional simulations of early structure formation show that the fraction of cold, dense gas available for accretion onto IMBHs or star formation exceeds $20 \%$ above $M_{h}=10^{6} M_{\odot}$ (Machacek et al. 2003). Furthermore, while radiative feedback (photodissociation and photoionization) from the progenitor massive star may initially quench $\mathrm{BH}$ accretion within the original host minihalo, new cold material will be readily available through the hierarchical merging of small gaseous subunits. In this section we assume that gas accretion onto BHs can actually occur with high efficiency in minihalos just above the cosmological Jeans mass.

As there is no compelling reason to expect the $m_{\mathrm{BH}}-\sigma_{*}$ relation to be set in primordial structures and be satisfied at the very high redshifts $(z \sim 15)$ considered in this work, we run two different sets of realizations. In the first we assume that in each major merger the hole in the main halo triples its mass via gas accretion, $\Delta m_{\mathrm{acc}}=2 m_{\mathrm{BH}}$, while in the second the hole accretes a fixed fraction of the host halo mass, $\Delta m_{\mathrm{acc}}=$ $10^{-3} M_{h}$. In both realizations this mass is added to the hole at the Eddington rate, $\dot{m}_{\mathrm{E}}=4 \pi G m_{p} m_{\mathrm{BH}} /\left(c \sigma_{\mathrm{T}} \epsilon\right)$, for a radiative efficiency $\epsilon=10 \%$. In the case of a merger in which only the satellite halo contains a $\mathrm{BH}$, the hole is placed at the center of the merger remnant after one dynamical friction timescale and must await a subsequent major merger event with a (smaller) 


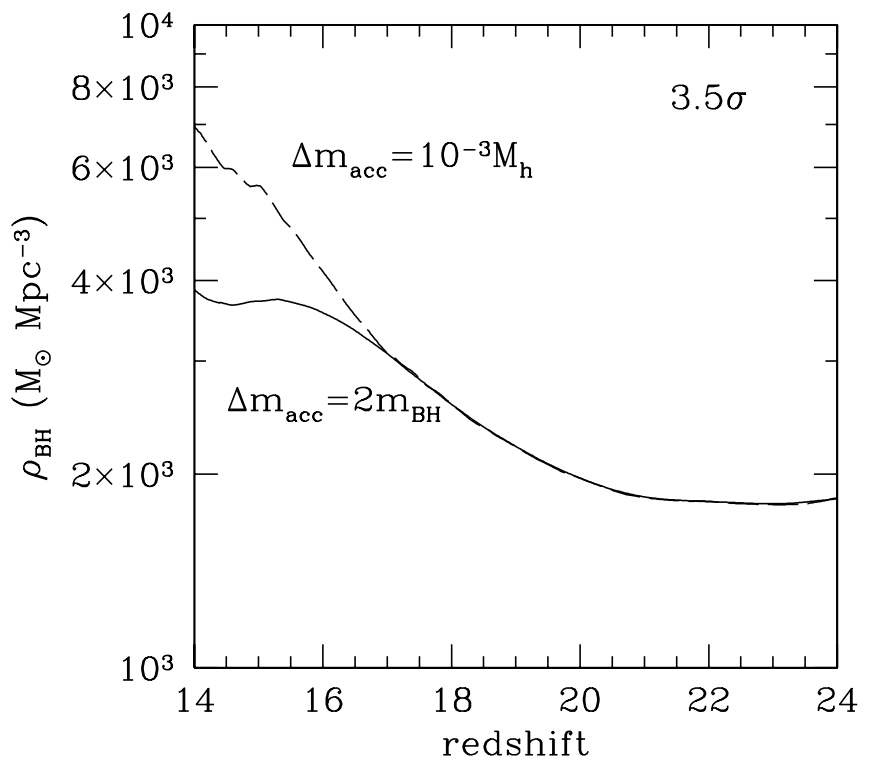

FIG. 2.-Comoving mass density in IMBHs as a function of redshift for the two different accretion recipes discussed in the text. Gas accretion onto BHs is assumed to occur in minihalos only after a major merger. Seed IMBHs form in minihalos collapsing at $z=24$ from $3.5 \sigma$ density peaks.

satellite to start accreting. Each accretion episode begins after one dynamical timescale (measured at radius $0.1 r_{\text {vir }}$ ) and typically lasts for a few Salpeter timescales, $t_{\mathrm{S}}=m_{\mathrm{BH}} / \dot{m}_{\mathrm{E}}=$ $\left(4.5 \times 10^{7} \mathrm{yr}\right)(\epsilon / 0.1)^{-1}$, until a mass $\Delta m_{\mathrm{acc}}$ has been added to the hole. Figure 2 shows how the comoving mass density of IMBHs builds up with cosmic time: with the adopted prescriptions, by a redshift of 14 this never exceeds $3 \%$ of the mass density of SMBHs inferred by Yu \& Tremaine (2002) in local galaxies.

\subsection{Miniquasars}

Assume now that a fraction $f_{\mathrm{UV}}$ of the bolometric power radiated by our Population III miniquasars is emitted as hydrogen-ionizing photons with mean energy $\langle h \nu\rangle$. For a given bolometric emissivity, the number of ionizing photons scales as $f_{\mathrm{UV}} /\langle h \nu\rangle$. One of the biggest uncertainties in discussing early reionization by miniquasars is their unknown emission spectrum. If the shape of the emitted spectrum from miniquasars followed the mean spectral energy distribution of the quasar sample in Elvis et al. (1994), then $f_{\mathrm{UV}}=0.3$ and $\langle h \nu\rangle=$ 3 ryd; hence, $f_{\mathrm{UV}} /\langle h \nu\rangle=0.1 \mathrm{ryd}^{-1}$. Miniquasars powered by IMBHs, however, are likely to be harder emitters than quasars. The hottest blackbody temperature in a Keplerian disk damping material onto a $\mathrm{BH}$ at the Eddington rate is $k T_{\max } \sim$ $(1 \mathrm{keV}) m_{\mathrm{BH}}^{-1 / 4}$ (Shakura \& Sunyaev 1973), where the hole mass is measured in solar mass units. The characteristic multicolor (cold) disk spectrum follows a power law with $L_{\nu} \sim$ $\nu^{1 / 3}$ at $h \nu<k T_{\max }$. Close to the epoch of reionization at $z \sim 15$, most IMBH holes will have masses in the range 200$1000 M_{\odot}$ (Fig. 3); hence, $k T_{\max } \sim 0.2-0.3 \mathrm{keV}$. The spectra of "ultraluminous" X-ray sources (ULXs; Colbert \& Mushotzky 1999) in nearby galaxies actually require both a soft and a hard component of comparable luminosities to describe the continuum emission. While the soft components are well fitted by cool multicolor disk blackbodies with $k T_{\text {max }} \simeq 0.15 \mathrm{keV}$, which may indicate IMBHs (e.g., Miller et al. 2003), the nonthermal power-law component has spectral slope $L_{\nu} \propto$ $\nu^{-\alpha}$, with $\alpha \approx 1$. A power law with slope $\alpha=1$ extending

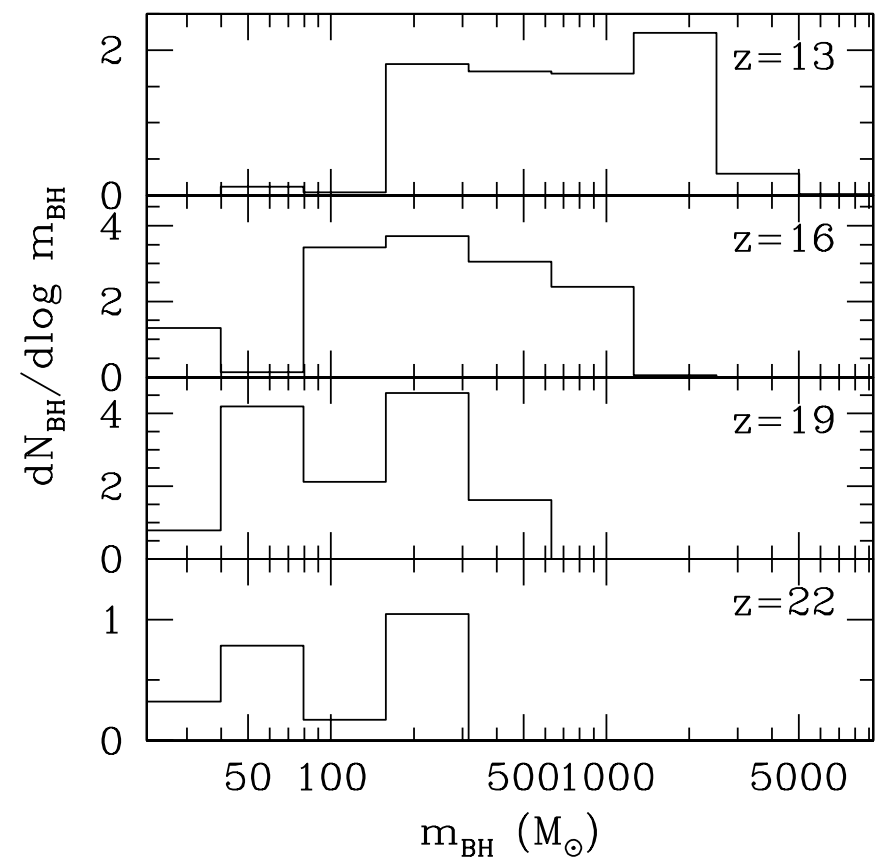

FIG. 3.- Mass function of accreting IMBHs predicted at four different redshifts by our fiducial model. Units are arbitrary. All seed holes are assumed to form at $z=24$ from $3.5 \sigma$ density peaks.

from $2 \mathrm{keV}$ down to $13.6 \mathrm{eV}$ has $f_{\mathrm{UV}}=1$ and $\langle h \nu\rangle=5$ ryd; hence, $f_{\mathrm{UV}} /\langle h \nu\rangle=0.2 \mathrm{ryd}^{-1}$. Figure 4 shows the cumulative number of ionizing photons produced per hydrogen atom for different values of $f_{\mathrm{UV}} /\langle h \nu\rangle$. A few ionizing hard photons per atom (less than for a stellar spectrum because of secondary ionizations and larger mean free path; see $\S 4$ ) should suffice to

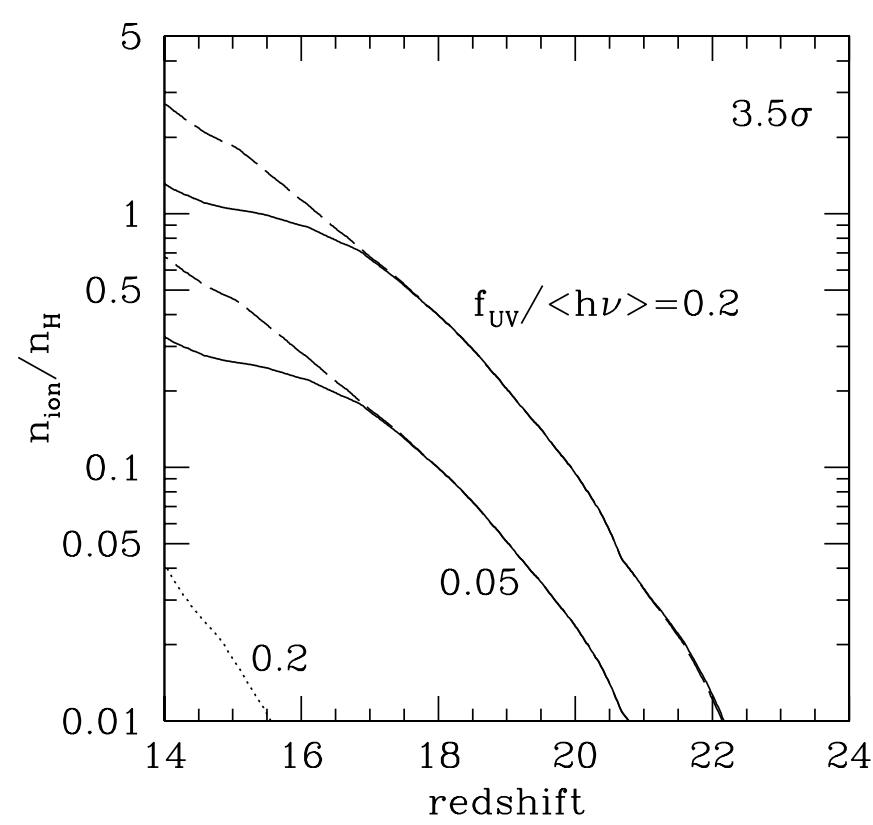

FIG. 4.-Cumulative number of ionizing photons per hydrogen atom produced by miniquasars, for different values of the fraction $f_{\mathrm{UV}}$ of the bolometric power that is emitted as radiation above 1 ryd with mean energy $\langle h \nu\rangle$ (in ryd). Solid curves: In each major merger the $\mathrm{BH}$ in the main halo accretes a mass $\Delta m_{\mathrm{acc}}=2 m_{\mathrm{BH}}$. Dashed curves: Same with $\Delta m_{\mathrm{acc}}=10^{-3} M_{h}$. Dotted curve: Same with $\Delta m_{\mathrm{acc}}=10^{-3} M_{h}, f_{\mathrm{UV}} /\langle h \nu\rangle=0.2 \mathrm{ryd}^{-1}$, but with gas accretion suppressed in minihalos with virial temperature $T_{\text {vir }} \lesssim 10^{4} \mathrm{~K}$. 
reionize the universe and keep the gas in overdense regions and filaments photoionized against radiative recombinations.

To illustrate the implications of these results, consider the following estimate for the number of $\mathrm{H}$-ionizing photons emitted by the initial population of progenitor massive stars. In our model the fraction, $f_{*}$, of cosmic baryons incorporated into Population III massive stars (progenitors of seed IMBHs) at $z=24$ is

$$
f_{*}=\frac{\left\langle m_{*}\right\rangle}{\left\langle m_{\mathrm{BH}}\right\rangle} \frac{\Omega_{\mathrm{BH}}}{\Omega_{b}} \approx 5 \times 10^{-7},
$$

where $\Omega_{b}$ is the baryon density parameter. Zero-metallicity stars in the range $40 M_{\odot}<m_{*}<500 M_{\odot}$ emit about 70,000 photons above 1 ryd per stellar baryon (Schaerer 2002). The total number of ionizing photons produced per baryon by progenitor Population III stars is then, using equation (1),

$$
\frac{n_{\text {ion }}}{n_{b}} \approx 70,000 f_{*} \approx 0.04
$$

well below what is needed to reionize the universe. Figure 4 shows that if $f_{\mathrm{UV}} /\langle h \nu\rangle$ is greater than $0.1 \mathrm{ryd}^{-1}$ and gas is accreted efficiently onto IMBHs, then miniquasars may be responsible for cosmological reionization at redshift $\sim 15$. We discuss in more detail some of the implications of reheating by hard radiation from miniquasars in $\S 4$.

\section{REDUCED ACCRETION EFFICIENCY AND DYNAMICS OF IMBH BINARIES}

Miniquasars powered by IMBHs forming in $3.5 \sigma$ peaks produce more ionizing photons than their progenitors and will reionize the IGM by $z \sim 15$. Such a conclusion is based on the assumption that IMBHs can grow rapidly via gas accretion during major mergers involving minihalos and shine in the EUV/soft X-rays. Local and global feedback effects, however, may act to reduce or even inhibit accretion in minihalos where atomic cooling is inefficient. More massive halos with $T_{\text {vir }}>$ $10^{4} \mathrm{~K}$, in which atomic cooling can operate, are expected to be very rare at these redshifts. If gas accretion was completely suppressed in minihalos with $T_{\mathrm{vir}} \lesssim 10^{4} \mathrm{~K}$, i.e., with masses below $10^{7.9}[(1+z) / 20]^{-3 / 2} M_{\odot}$, the lower emissivity would either shift the reionization epoch to lower redshift or make miniquasars a subdominant source of ionizing photons (Fig. 4), at least until halos massive enough that gas can initially cool and contract via excitation of hydrogen Ly $\alpha$ collapsed in large numbers.

Still, a viable scenario for early reionization by miniquasars may be one in which gas accretion onto IMBHs is much reduced compared to our fiducial model in $\S 2$, but seed holes are more numerous at the start in order to sustain the early production of ionizing radiation. BHs forming in $3 \sigma$ density fluctuations cannot be assumed to evolve in isolation, and the dynamics of $\mathrm{BH}$ binaries in shallow potential wells must be addressed. In this section we assess a model in which IMBHs populate all halos more massive than $M_{\text {seed }}=1.2 \times 10^{5} M_{\odot}$, corresponding to the $3 \sigma$ peaks of the primordial density field. In each major merger a mass $\Delta m_{\mathrm{acc}}=10^{-5} M_{h}$ (2 orders of magnitude smaller than in our fiducial model) is now added to the hole in the more massive halo at the Eddington rate; i.e., seed IMBHs do not grow appreciably as a result of gas accretion, and miniquasars are typically "on" only for a small fraction of the Salpeter timescale. Note that a high-accretion recipe with $\Delta m_{\mathrm{acc}}=10^{-3} M_{h}$ (say) is not viable in the case of

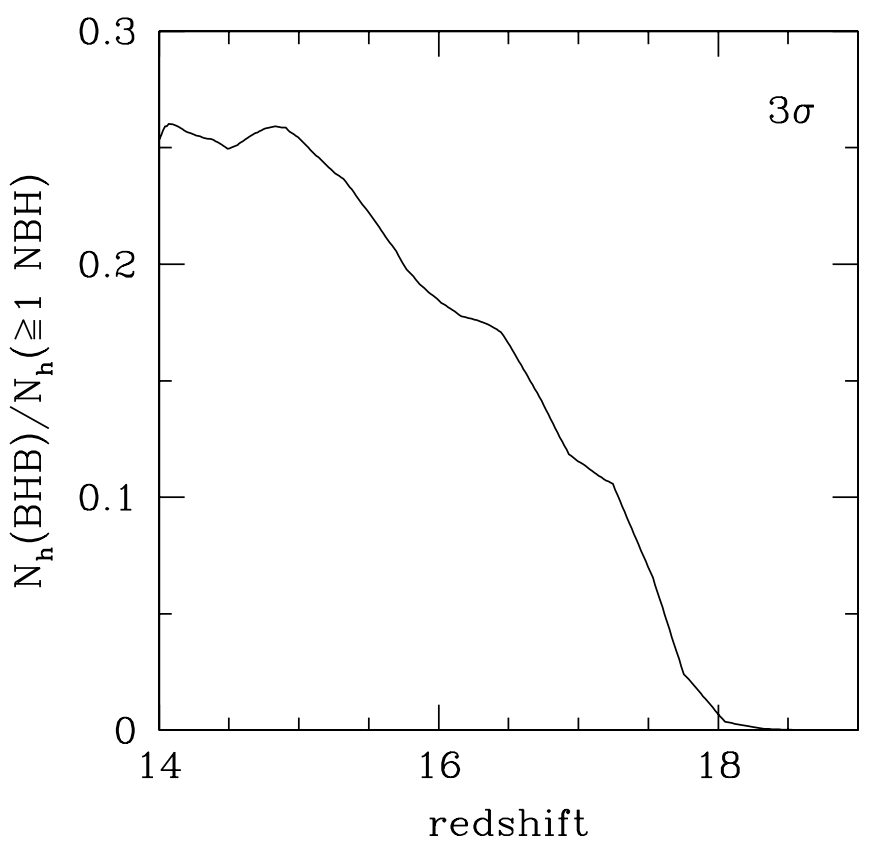

FIG. 5.-Fraction of all halos hosting nuclear black holes (NBHs) that contain a hard BH binary (BHB) system, as a function of redshift. Binaries are "hard" if their separation is $a \leq a_{h}$ (see text for details). Seed IMBHs form in minihalos collapsing at $z=24$ from $3 \sigma$ density peaks. In this figure we have assumed that BHBs do not shrink and coalesce (i.e., we set the hardening timescale $t_{h} \rightarrow \infty$ )

$3 \sigma$ seed holes, as it generates a $\rho_{\mathrm{BH}}$ that exceeds the mass density of SMBHs observed in nearby galaxies.

\subsection{IMBH Binaries}

As shown in Figure 5, if seed holes are as numerous as the 3 $\sigma$ density peaks, binary systems may form in significant numbers. The merging, driven by dynamical friction against the DM, of two minihalo plus IMBH systems with mass ratio $P \gtrsim 0.1$ will drag in the smaller hole toward the center, leading to the formation of a bound IMBH binary in the violently relaxed core of the newly merged system. In massive galaxies at low redshift, the subsequent evolution of the binary may be largely determined by the central stellar distribution. The binary initially shrinks ("hardens") by dynamical friction from distant stars acting on each $\mathrm{BH}$ individually, and later via threebody interactions, i.e., by capturing the stars that pass within a distance of the order of the binary semimajor axis and ejecting them at much higher velocities (Begelman, Blandford, \& Rees 1980). DM particles will be ejected by decaying binaries in the same way as the stars, i.e., through the gravitational slingshot. Their contribution to binary hardening will be approximately weighted by the fraction $f_{\mathrm{DM}}=\rho_{\mathrm{DM}} /\left(\rho_{\mathrm{DM}}+\rho_{*}\right)$, typically $\ll 1$ in massive galaxy cores.

In minihalos a numerous population of low-mass stars may be present if the IMF were bimodal, with a second peak at 1-2 $M_{\odot}$, as suggested by Nakamura \& Umemura (2001). Otherwise the binary will be losing orbital energy to the DM background. In the limit $f_{\mathrm{DM}} \gg 1$ (cf. Paper III), a binary with BH masses $m_{1} \geq m_{2}$ and semimajor axis $a(t)$ in an SIS dark halo of density $\rho$ and one-dimensional velocity dispersion $\sigma$ becomes "hard" when the binary separation falls below the value

$$
a_{h}=\frac{G m_{2}}{4 \sigma^{2}}=(0.04 \mathrm{pc})\left(\frac{m_{2}}{10^{3} M_{\odot}}\right) \sigma_{5}^{-2}
$$


(Quinlan 1996), where $\sigma_{5}$ is measured in units of $5 \mathrm{~km} \mathrm{~s}^{-1}$. The hardening of the binary modifies the density profile, removing mass interior to the binary orbit, depleting the galaxy core of DM, and slowing down further hardening. If $\mathcal{M}_{\mathrm{ej}}$ is the mass ejected by the $\mathrm{BH}$ pair, the binary evolution and its effect on galaxy cores are determined by two dimensionless quantities: the hardening rate

$$
H=\frac{\sigma}{G \rho} \frac{d a^{-1}}{d t}
$$

and the mass ejection rate

$$
J=\frac{1}{\left(m_{1}+m_{2}\right)} \frac{d \mathcal{M}_{\mathrm{ej}}}{d \ln a^{-1}} .
$$

The quantities $H$ and $J$ can be found from scattering experiments that treat the test particle-binary encounters one at a time (Quinlan 1996). We assume as in Papers II and III that the removal of matter creates a core of radius $r_{c}$ and constant density $\rho_{c} \equiv \rho\left(r_{c}\right)$, eroding a preexisting isothermal cusp with $\rho \propto r^{-2}$. The total mass ejected as the binary shrinks from $a_{h}$ to $a$ is then $\mathcal{M}_{\mathrm{ej}}=4 \sigma^{2} r_{c} / 3 G$, while the core radius grows as

$$
r_{c}(t)=\frac{3}{4 \sigma^{2}} G\left(m_{1}+m_{2}\right) \int_{a(t)}^{a_{h}} J(a) d a / a .
$$

The binary separation quickly falls below $r_{c}$, and subsequent evolution is slowed down as a result of the declining matter density, with a hardening time,

$$
t_{h}=\left|\frac{a}{\dot{a}}\right|=\frac{2 \pi r_{c}(t)^{2}}{H \sigma a},
$$

that becomes increasingly long as the binary shrinks. If hardening continues down to a separation

$$
a_{\mathrm{gr}}=(1 \mathrm{AU})\left[\frac{\left(m_{1}+m_{2}\right) m_{1} m_{2}}{10^{9.3} M_{\odot}^{3}}\right]^{1 / 4},
$$

the binary will coalesce within $200 \mathrm{Myr}$ (the Hubble time at $z=18$ ) as a result of the emission of gravitational waves. The pair must then manage to shrink by a factor $a_{h} / a_{\mathrm{gr}} \sim 5000$ for gravity wave emission to become efficient. Since the hardening and mass ejection rate coefficient are $H \approx 15$ and $J \approx 1$ in the limit of a very hard binary (Quinlan 1996), the hardening time in equation (8) can be rewritten (for $m_{1}=m_{2}$ ) as

$$
t_{h} \approx\left(1.3 \times 10^{5} \mathrm{yr}\right)\left(\frac{m_{2}}{10^{3} M_{\odot}}\right) \sigma_{5}^{-3} \frac{a_{h}}{a} \ln ^{2}\left(\frac{a_{h}}{a}\right) .
$$

This becomes longer than the then Hubble time already at $a_{h} / a \sim 100$. Note that for an equal-mass binary $a_{h} / a_{\mathrm{gr}} \propto$ $m_{2}^{1 / 4} \sigma^{-2}$. Thus, even if the $m_{\mathrm{BH}} \propto \sigma^{4}$ relation (Tremaine et al. 2002) were to hold at very high redshifts, for gravitational radiation to induce coalescence a hard $\mathrm{BH}$ binary must decay by a larger factor in minihalos with small velocity dispersion than in more massive galaxies. At these early epochs, when the $\mathrm{BH}$ occupation fraction is still small, we find that triple $\mathrm{BH}$ interactions are too rare to cause binaries to shrink (cf. Paper II). In the absence of any other mechanism for removing orbital angular momentum, the decay is then expected to stall at a separation much greater than $a_{\mathrm{gr}}$. This is even more true as it is the total matter density that was allowed to decrease in equation (7), not the density of low angular momentum particles that get close enough to extract energy from the binary; i.e., we have assumed that the "loss cone" is constantly refilled. Note that this would be a good assumption if orbital angular momentum losses to stars were the dominant mechanism of binary shrinkage, since the stellar relaxation timescale

$$
t_{r}=\frac{0.34 \sigma^{3}}{G^{2} m_{*} \rho_{c} \ln \Lambda}=150 \operatorname{Myr}\left(\frac{r_{c}}{0.5 \mathrm{pc}}\right)^{2}\left(\frac{4}{\ln \Lambda}\right) \sigma_{5}
$$

is typically much shorter than the hardening time in minihalos, and two-body scatterings will efficiently refill the loss cone.

\subsection{Gravitational Rocket and the Depletion of IMBHs from Host Minihalos}

The extent with which $\mathrm{BH}$ pairs lose angular momentum to DM particles or stars is one of the major uncertainties in computing merger timescales and makes it difficult to construct viable merger scenarios for BH binaries (e.g., Milosavljevic \& Merritt 2001). For illustrative purposes, we run here two different sets of realizations. In the first the hardening is due to the ejection of DM particles, the hardening timescale is long (from eqs. [8]-[10]), and binaries stall. Another possibility is that gas processes, rather than three-body interactions with stars or DM, may induce IMBH binaries to shrink rapidly and coalesce (e.g., Armitage \& Natarajan 2002; Gould \& Rix 2000). Gas in a system containing a hard binary will fall inward in just the same way as if there were a single hole at the center, as the binary separation is much smaller than the Bondi radius, $r_{\mathrm{B}}=G m_{\mathrm{BH}} / c_{\infty}^{2}=(0.5 \mathrm{pc})\left(m_{\mathrm{BH}} / 1000 M_{\odot}\right)(T / 1000 \mathrm{~K})^{-1}$. It will heat up and radiate away its binding energy. Some material will be accreted by each individual hole, while some will be flung out, removing binary orbital angular momentum and hastening the merger process. Further infall may replenish the gas reservoir on a dynamical timescale and keep the drag going. The details are unclear. As an extreme case we have therefore run a second set of realizations in which once a hard binary forms with separation $a_{h}$, it coalesces instantaneously. In the shallow potential wells of minihalos, the growth of IMBHs will then be halted by the gravitational rocket, the recoil due to the nonzero net linear momentum carried away by gravitational waves in the coalescence of two unequal-mass BHs. Radiation recoil is a strong field effect that depends on the lack of symmetry in the system and may eject IMBHs from the cores of minihalos. To date, the outcome of a gravitational rocket remains uncertain, as fully general relativistic numerical computations of radiation reaction effects are not available at the moment. The predicted recoil velocity, $v_{\mathrm{CM}}$, may be bracketed (Brandt \& Anninos 1999; Wiseman 1992) on the one side by quasi-Newtonian calculations predicting $v_{\mathrm{CM}}<$ $100 \mathrm{~km} \mathrm{~s}^{-1}$ (Fitchett 1983) and on the other by extrapolated perturbative results on test particles (Fitchett \& Detweiler 1984), allowing for rocket velocities up to $v_{\mathrm{CM}}=700 \mathrm{~km} \mathrm{~s}^{-1}$ for $m_{1}=1.2 m_{2}$ in a Schwarzschild geometry.

According to quasi-Newtonian calculations for circular orbits, while the binary shrinks its center of mass spirals outward with a velocity

$$
v_{\mathrm{CM}}=\left(1500 \mathrm{~km} \mathrm{~s}^{-1}\right)\left[\frac{f\left(m_{1} / m_{2}\right)}{f_{\max }}\right]\left(\frac{r_{\mathrm{S}}}{r_{\mathrm{ms}}}\right)^{4},
$$

where $r_{\mathrm{S}}=2 G\left(m_{1}+m_{2}\right) / c^{2}$ is the Schwarzschild radius of the system, $r_{\mathrm{ms}}=6 G m_{1} / c^{2}$ is the radius of the closest stable 


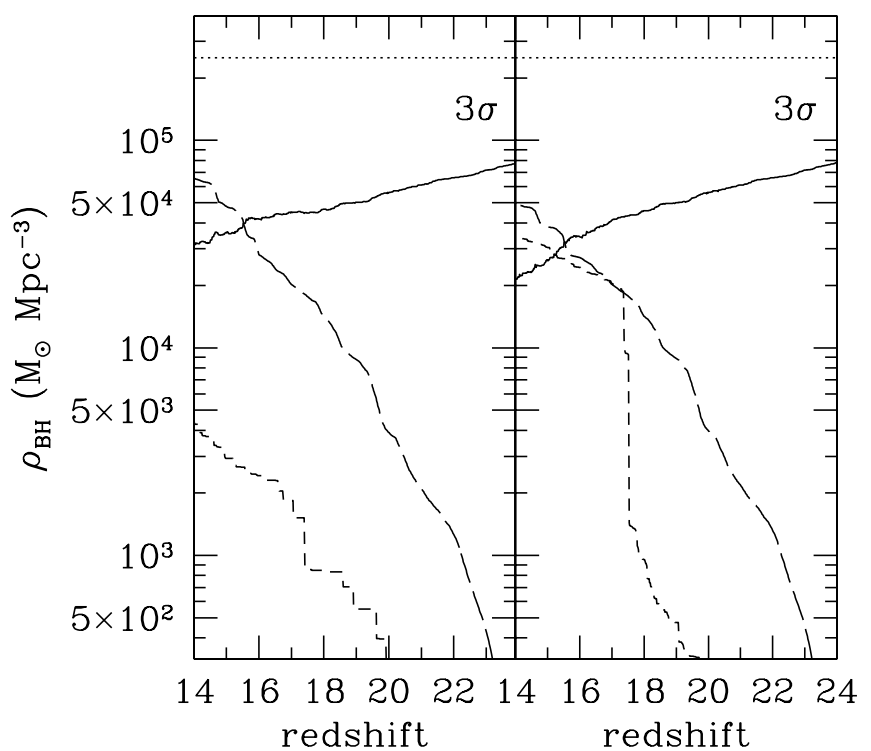

FIG. 6.-Contribution of nuclear, wandering, and intergalactic holes to the IMBH mass density as a function of redshift. In each major merger a mass $\Delta m_{\text {acc }}=10^{-5} M_{h}$ is accreted onto the $\mathrm{BH}$ in the main halo. Left: Model in which (most) binaries stall. Right: Model in which binaries shrink rapidly. Solid line: Mass density of IMBHs in galaxy nuclei. Long-dashed line: Wandering IMBHs retained in galaxy halos, mostly as a result of minor mergers. Short-dashed line: Intergalactic IMBHs ejected from the host after a gravitational rocket. Horizontal dotted line: Mass density of SMBHs in the nuclei of nearby galaxies inferred by Yu \& Tremaine (2002).

circular orbit for two nonrotating holes (Clark \& Eardley 1977), and the function

$$
f\left(\frac{m_{1}}{m_{2}}\right)=\left|\left(1+\frac{m_{1}}{m_{2}}\right)^{-2}\left(1+\frac{m_{2}}{m_{1}}\right)^{-3}\left(1-\frac{m_{2}}{m_{1}}\right)\right|
$$

reaches a maximum value $f_{\max }=0.01789$ for $m_{1}=2.6 m_{2}$. The extrapolated perturbative results (Fitchett \& Detweiler 1984) yield considerably higher recoil velocities for $m_{1}<4 m_{2}$, while for $m_{1}>7 m_{2}$ both calculations converge to recoil velocities below $15 \mathrm{~km} \mathrm{~s}^{-1}$. We adopt here the extrapolated perturbative results, as this assumption can be the most disruptive and lead to the larger modifications in terms of IMBH occupation fraction. The coalesced binary will leave the galaxy altogether if its recoil velocity exceeds the escape speed of the halo, giving origin to a population of intergalactic IMBHs. ${ }^{6}$

Figure 6 shows the predicted nuclear, wandering, and intergalactic IMBH mass density as a function of redshift, for the two sets of realizations described above. Two features are worth noting: the actual decrease with time of the density of nuclear IMBHs as more and more holes become wandering after minor mergers (cf. Fig. 2), and the 1 order of magnitude increase in the density of intergalactic holes due to the gravitational rocket after rapid coalescence. In the case where (most) binaries stall, the small population (in mass density) of intergalactic IMBHs is due to a few mergers involving nearequal-mass light binaries in the most massive halos, where the hardening timescale (eq. [10]) is shorter. In the model where binaries coalesce rapidly we find recoil velocities that exceed

${ }^{6}$ We have assumed that the final mass $m_{\mathrm{BH}}$ of the hole after coalescence satisfies the entropy-area relation (maximally efficient radiative merging): $m_{\mathrm{BH}}^{2}=m_{1}^{2}+m_{2}^{2}$. the escape speed from their hosts in $80 \%$ of the cases. Indeed, because of the rocket effect, the mass density of intergalactic holes exceeds that in the nuclear variety below a redshift of 15 or so.

The ejection into the IGM of IMBHs by the gravitational rocket effect appears, depending on the initial density of seed holes and the ability of BH binaries to shrink and coalesce, to decrease only slightly (by about 15\%) the "reionization efficiency" of miniquasars. This can be readily seen in Figure 7, which shows the cumulative number of $\mathrm{H}$-ionizing photons produced by miniquasars for the two cases ("binary stalling" vs. "instantaneous coalescence") discussed in this section. Even with a large reduction of the mass accreted by each $\mathrm{IMBH}$, the increased number of seeds produces more than a few ionizing photons per hydrogen atom by redshift 15 for a spectrum with $f_{\mathrm{UV}} /\langle h \nu\rangle \gtrsim 0.1 \mathrm{ryd}^{-1}$. If seed holes are as numerous as the $3 \sigma$ density peaks, the total number of Lyman continuum photons produced per baryon by their stellar progenitors is now

$$
\frac{n_{\text {ion }}}{n_{b}} \approx 70,000 f_{*} \approx 2.5
$$

i.e., 60 times larger than in the $3.5 \sigma$ case (cf. eq. [3]). In this case miniquasars may still dominate the ionizing photon budget for $f_{\mathrm{UV}} /\langle h \nu\rangle \gtrsim 0.2 \mathrm{ryd}^{-1}$ (or, of course, if $\left.\Delta m_{\mathrm{acc}}\right\rangle$ $\left.10^{-5} M_{h}\right)$.

\section{REIONIZATION BY HARD PHOTONS}

We have seen above that if cold gas in minihalos can be accreted either efficiently onto rare $(3.5 \sigma)$ Population III IMBHs or much less efficiently onto more numerous $(3 \sigma)$ seed holes, then miniquasars may be responsible for cosmological reionization at early times. In this section we discuss the reheating and reionization history of an IGM photoionized

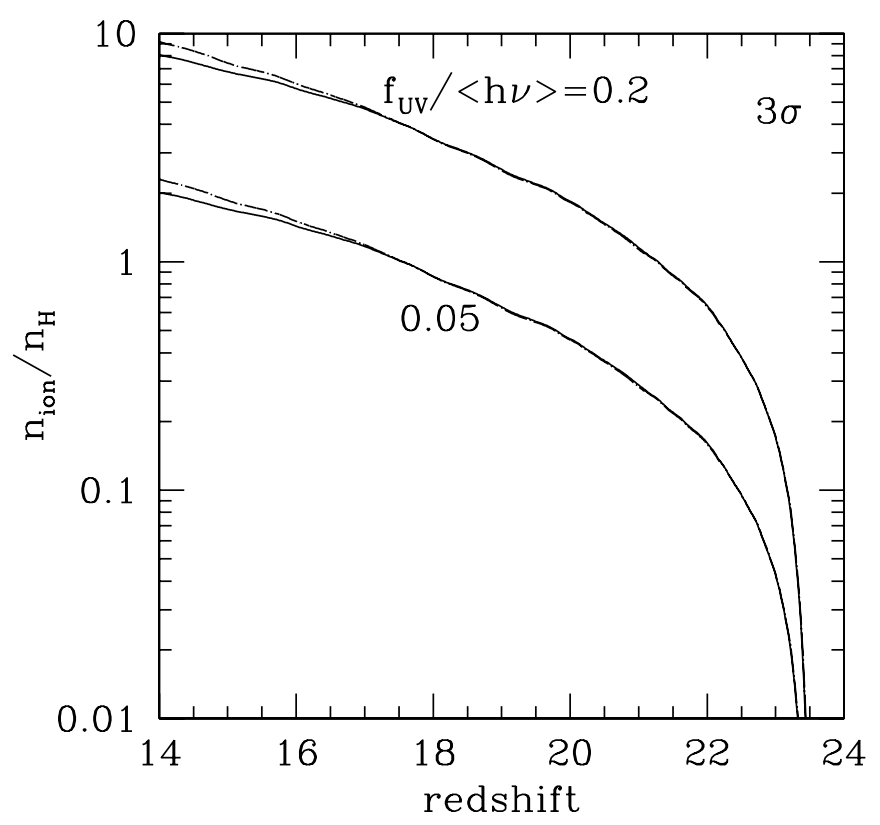

FIG. 7.-Cumulative number of ionizing photons per hydrogen atom produced by miniquasars for different values of $f_{\mathrm{UV}} /\langle h \nu\rangle$ (in units of $\mathrm{ryd}^{-1}$ ), assuming $\Delta m_{\mathrm{acc}}=10^{-5} M_{h}$. Seed IMBHs form in minihalos collapsing at $z=24$ from $3 \sigma$ density peaks. Dot-dashed curves: Model in which $\mathrm{BH}$ binaries stall. Solid curves: Same with rapid binary coalescence and gravitational rocket. 
primarily by a power-law metagalactic flux extending to X-ray energies, rather than by photons near the Lyman limit, as would be the case for purely stellar UV radiation. Reionization by energetic photons has been discussed recently by Venkatesan et al. (2001) and Oh (2001).

\subsection{Soft X-Ray Background Radiation}

Accretion onto IMBHs may be an attractive way to reionize the low-density IGM. A large fraction of the UV radiation from massive stars may not escape the dense sites of star formation or may be deposited locally in halo gas that recombines almost immediately. The harder radiation emitted from miniquasars is instead more likely to escape from the hosts into intergalactic space and may then produce more "durable" (albeit partial) ionization in the diffuse IGM.

In the pre-reionization universe, when the IGM is predominantly neutral, photons with energies above $24.6 \mathrm{eV}$ will be absorbed as they photoionize hydrogen or helium atoms. For a mixture of $\mathrm{H}$ and $\mathrm{He}$ with cosmic abundances, the effective bound-free absorption cross section ${ }^{7}$ can be approximated (to an accuracy of $30 \%$ in the range $50 \mathrm{eV}<h \nu<$ $2 \mathrm{keV})$ as $\sigma_{\mathrm{bf}} \approx\left(1.6 \times 10^{-17} \mathrm{~cm}^{2}\right)(h \nu / 1 \mathrm{ryd})^{-3}$. The continuum optical depth of a uniform IGM of hydrogen density $n_{0}(1+z)^{3}$, for photons emitted at frequency $\nu_{e}$ by a source at redshift $z_{e}$ beyond the redshift of reionization $z_{\text {rei }}$, is given by

$$
\tau=\int_{z_{\mathrm{rei}}}^{z_{e}} d z \frac{d l}{d z} n_{0}(1+z)^{3} \sigma_{\mathrm{bf}}(\nu) \approx 10^{6.4}\left(\frac{1 \mathrm{ryd}}{h \nu_{e}}\right)^{3}
$$

where $\nu \equiv \nu_{e}(1+z) /\left(1+z_{e}\right), d l / d z \simeq H_{0}^{-1} \Omega_{M}^{-1 / 2}(1+z)^{-5 / 2}$, and we have evaluated the integral from $z_{\text {rei }}=14$ to $z_{e}=19$. The universe is optically thick even to hard radiation, and all photons below $(h \nu)_{\text {thick }} \sim 2 \mathrm{keV}$ will be absorbed across a Hubble volume. Since a spectrum with $\nu L_{\nu} \sim$ const (like the nonthermal component observed in ULXs) is characterized by equal power per logarithmic frequency interval, photoelectric absorption by the host galaxies will not significantly attenuate the ionizing energy flux.

The mean free path of ionizing photons in the neutral IGM is

$$
\lambda=\frac{1}{n_{0}(1+z)^{3} \sigma_{\mathrm{bf}}} \approx(13 \mathrm{pc})\left(\frac{1+z}{20}\right)^{-3}\left(\frac{h \nu}{1 \mathrm{ryd}}\right)^{3} .
$$

At $z=24$, the comoving space density of halos collapsing from $3.5 \sigma$ density peaks is $\sim 15 \mathrm{Mpc}^{-3}$, corresponding to a mean proper distance between neighboring halos of $15 \mathrm{kpc}$. Photons with energies above $(h \nu)_{\text {overlap }} \sim 150 \mathrm{eV}$ will then have a mean free path greater than the mean separation between sources. We can therefore separate the radiation field into two components: a fluctuating EUV field in the range $13.6 \mathrm{eV}<h \nu<150 \mathrm{eV}, J_{\mathrm{UV}}$, which creates expanding patchy $\mathrm{H}$ II regions, and a nearly uniform soft $X$-ray background component in the range $150 \mathrm{eV}<h \nu<2 \mathrm{keV}, J_{\mathrm{X}}$, which partially ionizes the IGM in a fairly homogeneous manner.

\subsection{Secondary Ionizations}

Prior to the epoch of reionization breakthrough, before the overlapping of fully ionized (by EUV photons) bubbles around individual miniquasars, soft X-ray radiation will

\footnotetext{
7 The exact form of the photoionization cross section for $\mathrm{H}$ I was taken from Spitzer (1978), and we have used the fit from Verner et al. (1996) for He I (see also Miralda-Escudé 2000).
}

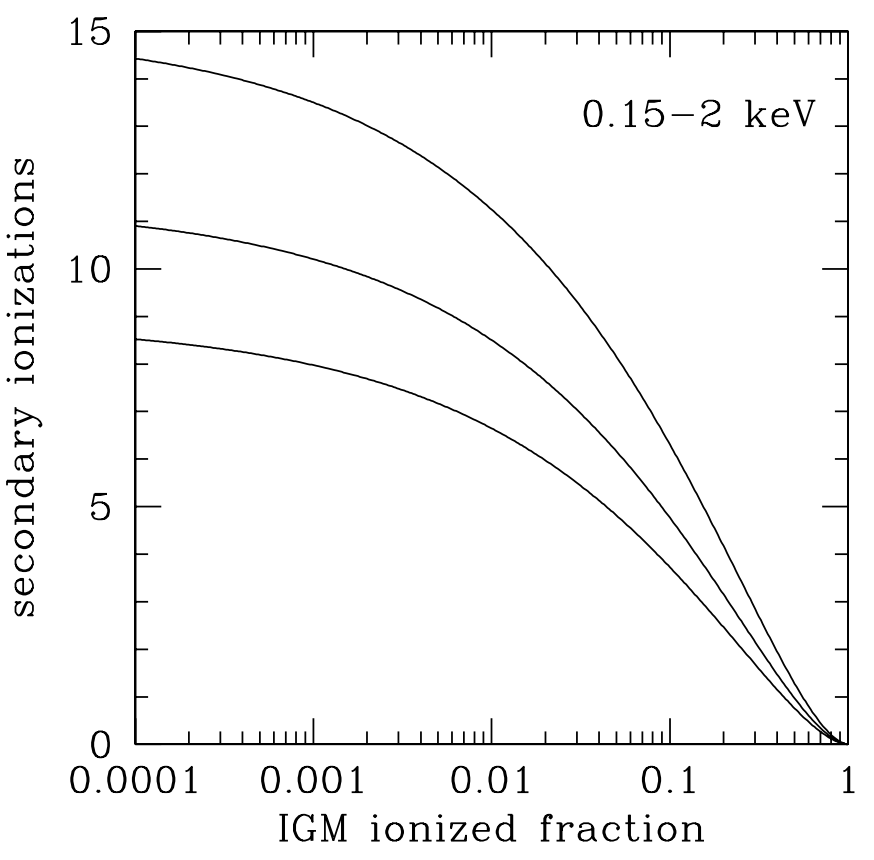

FIG. 8.-Number of $\mathrm{H}$ secondary ionizations per ionizing photon vs. IGM ionized fraction. A power-law radiation field with energy spectrum $J_{\mathrm{X}} \propto \nu^{-\alpha}$, extending from $150 \mathrm{eV}$ to $2 \mathrm{keV}$, has been assumed. The three curves from top to bottom are for $\alpha=0.5,1$, and 1.5, respectively. As the medium becomes more ionized, an increasing fraction of energy is deposited as heat.

produce a warm $\left(T_{\mathrm{IGM}} \sim\right.$ few $\left.\times 100-1000 \mathrm{~K}\right)$, weakly ionized $(x \sim 0.1)$ IGM (Venkatesan et al. 2001). X-rays alone do not produce a fully ionized medium but can partially photoionize the gas by repeated secondary ionizations. A primary nonthermal photoelectron of energy $E=1 \mathrm{keV}$ in a medium with residual ionization (from the recombination epoch) $x=$ $2 \times 10^{-4}$ will create over two dozen secondary electrons, depositing a fraction $f_{\text {ion }} \approx 37 \%$ of its initial energy as secondary ionizations of hydrogen and only $f_{\text {heat }} \approx 13 \%$ as heat (Shull \& van Steenberg 1985).

Figure 8 shows the mean number of secondary ionizations per ionizing photon as a function of IGM ionized fraction, for a power-law spectrum extending from $150 \mathrm{eV}$ to $2 \mathrm{keV}$ ("uniform soft X-ray background"). The calculation uses the analytic fits given by Shull \& van Steenberg (1985). The timescale for electron-electron encounters resulting in a fractional energy loss $f=\Delta E / E$,

$$
t_{e e} \approx(140 \mathrm{yr}) E f\left(\frac{1+z}{20}\right)^{-3}\left(\frac{\ln \Lambda}{20}\right)^{-1} x^{-1}
$$

(where $E$ is measured in $\mathrm{keV}$ ), is typically much shorter that the electron Compton cooling timescale off cosmic microwave background $(\mathrm{CMB})$ photons, $t_{\mathrm{C}}=\left(7 \times 10^{6} \mathrm{yr}\right)[(1+z) / 20]^{-4}$, and thus the primary photoelectron will ionize and heat the surrounding medium before it is cooled by the CMB.

The history of reheating and reionization by a hard $\nu^{-1}$ spectrum may then be described as follows. Initially, when the IGM is mostly neutral, each photoionization produces a host of secondary collisional ionizations, with about one hydrogen secondary ionization for every $37 \mathrm{eV}$ of energy in the primary photoelectron. For photon energies above $0.2 \mathrm{keV}$, the ratio of the $\mathrm{H}$ I/He I photoionization cross sections drops below $4 \%$ : since the primordial ratio of helium to hydrogen is about $8 \%$, the photoionization and heating rates will be dominated by 
helium absorption, which exceeds the hydrogen contribution by $\sim 2: 1$. While $\mathrm{He}_{\mathrm{I}}$ is the main source of hot primary photoelectrons, however, $\mathrm{H}$ I undergoes the bulk of secondary ionizations. Secondary ionizations operate efficiently for $\mathrm{H}_{\mathrm{I}}$ and $\mathrm{He}$ I, but not for He II (Shull \& van Steenberg 1985). Because of losses to secondary ionizations and to collisional excitations of $\mathrm{H}$ I and $\mathrm{He}$ I before thermalization, the heating by energetic photons is initially not as efficient as for photons near the photoelectric thresholds. The amount of heat deposited into a unit proper volume of the IGM by the mean field can be written in first approximation as

$$
\Delta Q=f_{\text {heat }} \frac{4 \pi}{c} \int_{\nu_{\text {overlap }}}^{\nu_{\text {thick }}} d \nu J_{\mathrm{X}}=(1+z)^{3} f_{\text {heat }} f_{\mathrm{X}} \epsilon \rho_{\mathrm{acc}} c^{2},
$$

where $f_{\mathrm{X}}$ is the fraction of the bolometric emissivity of miniquasars that is radiated between $\nu_{\text {overlap }}$ and $\nu_{\text {thick}}, f_{\mathrm{X}}=$ $\ln \left(\nu_{\text {thick }} / \nu_{\text {overlap }}\right) / \ln \left(\nu_{2} / \nu_{1}\right)$ for a $\nu^{-1}$ spectrum extending from $\nu_{1}$ to $\nu_{2}$. For the spectrum we have chosen, the result is only logarithmically sensitive to the integration bounds, and we set $f_{\mathrm{X}}=0.4$. In the scenarios for the formation and growth of IMBHs discussed in the previous sections, gas accretion onto IMBHs rapidly builds up (by $z \sim 22$ ) a comoving mass density $\rho_{\text {acc }}$ in excess of a few hundred $M_{\odot} \mathrm{Mpc}^{-3}$, enough to heat up the gas to initial temperatures:

$$
T=\frac{\Delta Q}{3 / 2 n k} \approx(1500 \mathrm{~K})\left(\frac{f_{\text {heat }}}{0.13}\right)\left(\frac{\rho_{\mathrm{acc}}}{200 \mathrm{M}_{\odot} \mathrm{Mpc}^{-3}}\right) .
$$

This same radiation field would ionize the IGM to $x \approx 4 \times 10^{-3}\left(f_{\text {ion }} / 0.37\right)\left(\rho_{\text {acc }} / 200 M_{\odot} \mathrm{Mpc}^{-3}\right)$. Since both the hydrogen recombination and the Compton cooling time are much longer than the then Hubble time for $x<0.1$, the gas evolves adiabatically. Once the IGM ionized fraction increases to $x \approx 0.1$, the number of secondary ionizations per ionizing photon drops to a few (Fig. 8), and the bulk of the primary's energy goes into heat $\left(f_{\text {heat }} \approx 0.6\right)$ via elastic Coulomb collisions with thermal electrons. At this point Compton cooling will restrict any further increase in temperature: it is the rate of direct photoionizations by UV photons in the soft end of the spectrum that drives the final stages of the reionization process, until the fully ionized bubbles associated with individual sources finally overlap.

As $\mathrm{H}$ I and $\mathrm{He}_{\mathrm{I}}$ reionization nears completion, the only means for a hard radiation field to inject energy into the plasma is via He II photoionization and Compton heating. Compton heating is much less efficient than He II photoionization heating: the small Thompson cross section implies that the universe is always optically thin to Compton heating of electrons. Furthermore, the energy exchange per interaction is only a small fraction of the photon energy, $\sim h \nu\left(h \nu / m_{e} c^{2}\right)$. Thus, in most situations, Compton heating will play a subdominant role compared to He II photoheating (cf. Madau \& Efstathiou 1999).

\subsection{Entropy Floor}

As recently pointed out by Oh \& Haiman (2003), soft $\mathrm{X}$-rays can exert another important effect on the IGM and subsequent star formation, as the warm, partially ionized medium produced by energetic photons has a high entropy. Gas at the mean density that is heated to temperatures

$$
T_{\text {IGM }}>(90 \mathrm{~K})\left(\frac{T_{\text {vir }}}{3000 \mathrm{~K}}\right)\left(\frac{\delta}{200}\right)^{-2 / 3}
$$

(where $\delta$ is the overdensity of the gas in the minihalo in the absence of preheating) will have entropy in excess of that acquired by gravitational shock heating alone. It will therefore have excess pressure after adiabatic heating and compression. As shown in the previous section, such temperatures are easily achievable with miniquasars. This entropy floor (1) greatly reduces gas clumping, curtailing the number of photons needed to maintain reionization, and (2) results in significantly lower gas densities in the cores of minihalos that suppress rapid $\mathrm{H}_{2}$ formation. The latter effect may imply that X-rays inhibit rather than enhance star formation (Oh \& Haiman 2003).

An entropy floor could conceivably also reduce the efficiency with which miniquasars can shine. In halos that have had their central densities reduced and are unable to form stars, IMBHs may still accrete gas, but at a considerably reduced rate. Miniquasars, however, are $\sim 15-150$ times more efficient at producing ionizing radiation per processed baryon than metal-free stars and thus need a much smaller reservoir of cold gas to reionize the universe. If $f_{\mathrm{UV}} /\langle h \nu\rangle \sim$ 0.2 and $100 \mathrm{MeV}$ are released per baryon, then only $\sim 7 \times 10^{-7}$ of all baryons need to be accreted onto IMBHs to provide one ionizing photon per baryon, compared to $\sim 10^{-5}$ for massive metal-free stars (Bromm et al. 2001) and $\sim 10^{-4}$ for metal-free stars with a Salpeter IMF (Tumlinson \& Shull 2000). The cold gas that cooled prior to the establishment of the entropy floor (and formed an accretion disk around the central $\mathrm{BH}$ ) will still be available as fuel. If only a fraction $f_{\text {cool }} \sim 1 \%$ of the gas in a $3.5 \sigma$ peak halo cooled to form an accretion disk around the central $\mathrm{BH}$ before an entropy floor was established, this still represents $\sim 5 \times$ $10^{-6}\left(f_{\text {cool }} / 0.01\right)$ of all baryons, enough to provide 10 ionizing photons per hydrogen atom; this number increases by an order of magnitude if $3 \sigma$ peaks are involved instead. By contrast, these baryon fractions are insufficient to reionize the universe with Population III metal-free stars; the entropy floor would prohibit full reionization by such objects. The combination of X-rays (which reduce gas clumping and thus the number of UV photons required to complete reionization) and UV photons (which carry reionization to completion) could allow miniquasars to reionize the universe quite efficiently.

\section{SUMMARY}

This paper should be read as a first attempt at treating the impact on the very early IGM of Population III IMBHs - the remnants of the first generation of massive stars - in the context of hierarchical structure formation theories. We have tried to incorporate some of the essential astrophysics into a scenario for the reheating and reionization of the universe by miniquasars. In our model quasar activity is driven by major mergers and IMBHs are able to accrete at the Eddington rate only in the densest inner regions of the merger remnant. If seed IMBHs are as rare as the $3.5 \sigma$ peaks of the primordial density field, they evolve largely in isolation; a significant number of BH binary systems form if IMBHs populate the more numerous $3 \sigma$ peaks instead. In the case of rapid binary coalescence, rather than accrete and shine as miniquasars, a fraction of IMBHs will be displaced from galaxy centers and ejected into the IGM by the gravitational rocket effect. Note that, for gravitational radiation to induce coalescence, a hard $\mathrm{BH}$ binary must decay by a larger factor in minihalos with small velocity dispersion than in more massive galaxies. The loss of orbital angular momentum to a gaseous disk, rather 
than via three-body interactions with DM particles or stars, may drive IMBH binaries to merge rapidly, but the details are unclear.

Under a number of different assumptions for the amount of gas accreted onto IMBHs and their emission spectrum, miniquasars powered by IMBHs, and not their metal-free stellar progenitors, may be responsible for cosmological reionization at $z \sim 15$. The WMAP data on $\tau_{e}$ may then be setting constraints on gas accretion onto the earliest generation of IMBHs, as well as on the IMF of the first burst of star formation in the universe. Even if the "UV radiation efficiency" of miniquasars was lower and Population III stars were the dominant source of ionizing radiation at $z \sim 25$, the transition from Population III to Population II at a redshift possibly determined by the level of metal enrichment of the IGM would decrease by more than an order of magnitude the emission rate of Lyman continuum photons (e.g., Cen 2003a). Hard radiation from miniquasars could then sustain ionization in the redshift range $z_{\text {rei }}<z<20$ (say), before ordinary stars with a Salpeter IMF took over.

At $z \gtrsim 4-5$, the known population of quasars appears not to contribute significantly to the hydrogen-ionizing background (Madau, Haardt, \& Rees 1999). The comoving space density of luminous SDSS quasars at $z \sim 6$ is 20 times smaller than that at $z \sim 3$ (Fan et al. 2003). The dearth of bright QSOs at $z \sim 6$ may be reconciled with the existence of a substantial population of accreting IMBHs at $z \sim 15$ if there were two distinct periods of quasar activity. The first may have ended with the demise of miniquasars due to radiative feedback mechanisms after the reionization epoch (as photoionization from background UV photons unbinds the gas in the shallow potential wells of minihalos; Barkana \& Loeb 1999; Haiman, Abel, \& Madau 2001) or to a raised entropy floor. Efficient gas accretion onto nuclear BHs could conceivably start again only at $z \lesssim 10<z_{\text {rei }}$, in $T_{\text {vir }} \gg 10^{4} \mathrm{~K}$ halos farther down in the merger hierarchy that are not susceptible to Jeans smoothing and gas cooling suppression effects. This may lead to a second "quasar era" seeded by more massive $\left(\sim 5000 M_{\odot}\right)$ preexisting holes.

One might worry that with a sufficiently hard ionizing spectrum H I and He II $\rightarrow$ He III reionization may be simultaneous, whereas observations of the $\mathrm{He}$ II Ly $\alpha$ forest have been interpreted as evidence that the double reionization of helium occurred at a redshift of $\sim 3$ (e.g., Kriss et al. 2001; Reimers et al. 1997). For a $\nu^{-1}$ miniquasar spectrum there are 3 times more He II-ionizing photons ( $h \nu>4$ ryd) per He atom than photons above 1 ryd per $\mathrm{H}$ atom. As doubly ionized helium recombines about 6 times faster than hydrogen, the number of greater than 4 ryd photons per He atom emitted in one recombination timescale is only half of the corresponding value for hydrogen, and there will be just a small delay between the complete overlapping of $\mathrm{H}$ II and $\mathrm{He}$ III regions (Miralda-Escudé \& Rees 1993; Madau \& Meiksin 1994). He II reionization by miniquasars at $z \gtrsim 10$ would provide a significant boost to the gas temperature, with interesting implications for the thermal history of the IGM (e.g., Hui \& Haiman 2003). The patchy He II absorption observed at $z \sim 3$ could then be explained by variations in the spectra of the ionizing sources (Smette et al. 2002) or may imply that He II was reionized twice: a first time by miniquasars, followed by recombination as Population II stars with softer spectra took over, and then by a second overlap phase at $z \sim 3$ during the second quasar epoch.

Absorption in the intervening IGM will make miniquasars at $z \sim 15$ inaccessible to direct observations from $2 \mu \mathrm{m}$ down to soft X-ray energies. He II recombination lines longward of H I Ly $\alpha$ may be potentially detectable by the James Webb Space Telescope only from the brightest miniquasars $(\mathrm{Oh}$, Haiman, \& Rees 2001a). An alternative way to probe the end of the cosmic "dark ages" and discriminate between different reionization histories is through $21 \mathrm{~cm}$ tomography of neutral hydrogen (Madau, Meiksin, \& Rees 1997). In general, $21 \mathrm{~cm}$ spectral features will display angular structure, as well as structure in redshift space due to inhomogeneities in the gas density field, hydrogen ionized fraction, and spin temperature. As recently discussed by Tozzi et al. (2000), Ciardi \& Madau (2003), and Furlanetto, Sokasian, \& Hernquist (2004), the fluctuations in $21 \mathrm{~cm}$ line induced by the "cosmic web" that develops at early times in CDM-dominated cosmologies could be detected in emission against the CMB provided that (1) at epochs when the IGM is still mainly neutral, the first generation of (Population III) stars produce enough UV continuum photons with energies between 10.2 and $13.6 \mathrm{eV}$ to mix the hyperfine levels and decouple the hydrogen spin temperature from $T_{\mathrm{CMB}}$; and (2) the IGM is "warm," i.e., $T_{\mathrm{IGM}} \gg T_{\mathrm{CMB}}=$ $(57 \mathrm{~K})[(1+z) / 20]$. X-ray radiation naturally produces a warm, weakly ionized IGM. Miniquasars turning on at early stages may then make the IGM visible in $21 \mathrm{~cm}$ emission as structure develops, before the universe is actually reionized. Brightness temperature fluctuations may be detectable by future facilities like the Low Frequency Array (LOFAR) if foreground contamination from unresolved extragalactic point sources (Di Matteo et al. 2002; Oh \& Mack 2003) can be successfully removed by using spectral structure in frequency space.

Finally, we remark that accreting IMBHs may release large amounts of energy to their environment in other forms than radiative output. Outflows from miniquasars may inject kinetic energy into intergalactic space and raise the temperature of the IGM to a much higher adiabat than expected from photoionization. In the type of scenario discussed in $\S 3$, gas accretion along cosmic history builds up by $z \sim 14$ a comoving mass density, $\rho_{\text {acc }}$, in excess of $10^{4} M_{\odot} \mathrm{Mpc}^{-3}$. If a fraction $f_{w}$ of the accreted rest-mass energy was used to drive an outflow and ultimately deposited into the IGM, the energy input per baryon at this epoch,

$$
E_{w}=f_{w} \frac{\rho_{\mathrm{acc}} c^{2}}{n_{b}} \sim(0.1 \mathrm{keV})\left(\frac{f_{w}}{0.05}\right)\left(\frac{\rho_{\mathrm{acc}}}{10^{4} M_{\odot} \mathrm{Mpc}^{-3}}\right)
$$

would suffice to preheat vast regions of the universe to temperatures above a few $\times 10^{5} \mathrm{~K}$ and so inhibit the formation of early dwarf galaxies (Benson \& Madau 2003). The detailed thermal history of the IGM may then depend on different forms of "feedback" mechanisms from miniquasars during the epoch of galaxy formation.

Support for this work was provided by NASA grant NAG511513 and NSF grant AST 02-05738 (P. M.), by grant MIUR COFIN2002 (F. H.), and by the Royal Society (M. J. R.). 
Abel, T., Bryan, G. L., \& Norman, M. L. 2002, Science, 295, 93 Armitage, P. J., \& Natarajan, P. 2002, ApJ, 567, L9 Baraffe, I., Heger, A., \& Woosley, S. E. 2001, ApJ, 550, 890 Barkana, R., \& Loeb, A. 1999, ApJ, 523, 54

Begelman, M. C., Blandford, R. D., \& Rees, M. J. 1980, Nature, 287, 307 Benson, A. J., \& Madau, P. 2003, MNRAS, 344, 835

Bond, J. R., Arnett, W. D., \& Carr, B. J. 1984, ApJ, 280, 825

Brandt, S., \& Anninos, P. 1999, Phys. Rev. D, 60, 4005

Bromm, V., Coppi, P. S., \& Larson, R. B. 2002, ApJ, 564, 23

Bromm, V., Kudritzki, R. P., \& Loeb, A. 2001, ApJ, 552, 464

Cavaliere, A., \& Vittorini, V. 2000, ApJ, 543, 599

Cen, R. 2003a, ApJ, 591, 12 2003b, ApJ, 591, L5

Ciardi, B., Ferrara, A., \& White, S. D. M. 2003, MNRAS, 344, L7

Ciardi, B., \& Madau, P. 2003, ApJ, 596, 1

Clark, J. P., \& Eardley, D. M. 1977, ApJ, 215, 311

Colbert, E. J. M., \& Mushotzky, R. F. 1999, ApJ, 519, 89

Colpi, M., Mayer, L., \& Governato, F. 1999, ApJ, 525, 720

Di Matteo, T., Perna, R., Abel, T., \& Rees, M. J. 2002, ApJ, 564, 576

Djorgovski, S. G., Castro, S., Stern, D., \& Mahabal, A. A. 2001, ApJ, 560, L5 Elvis, M., et al. 1994, ApJS, 95, 1

Fabian, A. 2003, in Carnegie Observatories Astrophysics Series, Vol. 1:

Coevolution of Black Holes and Galaxies, ed. L. C. Ho (Cambridge:

Cambridge Univ. Press), in press (astro-ph/0304122)

Fan, X., et al. 2002, AJ, 123, 1247 2003, AJ, 125,1649

Ferrarese, L. 2002, ApJ, 578, 90

Ferrarese, L., \& Merritt, D. 2000, ApJ, 539, L9

Fitchett, M. 1983, MNRAS, 203, 1049

Fitchett, M., \& Detweiler, S. 1984, MNRAS, 211, 933

Fuller, T. M., \& Couchman, H. M. P. 2000, ApJ, 544, 6

Furlanetto, S. R., Sokasian, A., \& Hernquist, L. 2004, MNRAS, 347, 187

Gebhardt, K., et al. 2000, ApJ, 543, L5

Ghigna, S., Moore, B., Governato, F., Lake, G., Quinn, T., \& Stadel, J. 1998 , MNRAS, 300, 146

Glover, S. C. O., \& Brand, P. W. J. L. 2003, MNRAS, 340, 210

Gould, A., \& Rix, H. 2000, ApJ, 532, L29

Haiman, Z., Abel, T., \& Madau, P. 2001, ApJ, 551, 599

Haiman, Z., Abel, T., \& Rees, M. J. 2000, ApJ, 534, 11

Haiman, Z., \& Holder, G. P. 2003, ApJ, 595, 1

Heger, A., \& Woosley, S. E. 2002, ApJ, 567, 532

Hui, L., \& Haiman, Z. 2003, ApJ, 596, 9

Islam, R. R., Taylor, J. E., \& Silk, J. 2003, MNRAS, 340, 647

Kauffmann, G., \& Haehnelt, M. G. 2000, MNRAS, 311, 576

Kogut, A., et al. 2003, ApJS, 148, 161

Kriss, G. A., et al. 2001, Science, 293, 1112

Kudritzki, R. P. 2002, ApJ, 577, 389

Machacek, M. M., Bryan, G. L., \& Abel, T. 2001, ApJ, 548, 509 2003, MNRAS, 338, 273
Madau, P., \& Efstathiou, G. 1999, ApJ, 517, L9

Madau, P., Haardt, F., \& Rees, M. J. 1999, ApJ, 514, 648

Madau, P., \& Meiksin, A. 1994, ApJ, 433, L53

Madau, P., Meiksin, A., \& Rees, M. J. 1997, ApJ, 475, 429

Madau, P., \& Rees, M. J. 2001, ApJ, 551, L27 (Paper I)

McClintock, J. E., \& Remillard, R. A. 2003, preprint (astro-ph/0306213)

Mihos, J. C., \& Hernquist, L. 1996, ApJ, 464, 641

Miller, J. M., Fabbiano, G., Miller, M. C., \& Fabian, A. C. 2003, ApJ, 585, L37

Milosavljevic, M., \& Merritt, D. 2001, ApJ, 563, 34

Miralda-Escudé, J. 2000, ApJ, 528, L1

Miralda-Escudé, J., \& Rees, M. J. 1993, MNRAS, 260, 617

Nakamura, F., \& Umemura, M. 2001, ApJ, 548, 19

Oh, S. P. 2000, Ph.D. thesis, Princeton Univ. 2001, ApJ, 553, 499

Oh, S. P., \& Haiman, Z. 2003, MNRAS, 346, 456

Oh, S. P., Haiman, Z., \& Rees, M. J. 2001a, ApJ, 553, 73

Oh, S. P., \& Mack, K. J. 2003, MNRAS, 346, 871

Oh, S. P., Nollett, K. M., Madau, P., \& Wasserburg, G. J. 2001b, ApJ, 562, L1

Quinlan, G. D. 1996, NewA, 1, 35

Redmount, I. H., \& Rees, M. J. 1989, Comments Astrophys., 14, 165

Reimers, D., Köhler, S., Wisotzki, L., Groote, D., Rodriguez-Pascual, P., \&

Wamsteker, W. 1997, A\&A, 327, 890

Schaerer, D. 2002, A\&A, 382, 28

Schneider, R., Ferrara, A., Natarajan, P., \& Omukai, K. 2002, ApJ, 571, 30

Shakura, N. I., \& Sunyaev, R. A. 1973, A\&A, 24, 337

Shull, J. M., \& van Steenberg, M. E. 1985, ApJ, 298, 268

Smette, A., Heap, S. R., Williger, G. M., Tripp, T. M., Jenkins, E. B., \& Songaila, A. 2002, ApJ, 564, 542

Sokasian, A., Abel, T., Hernquist, L., \& Springel, V. 2003, MNRAS, 344, 607

Somerville, R. S., \& Livio, M. 2003, ApJ, 593, 611

Spergel, D. N., et al. 2003, ApJS, 148, 175

Spitzer, L. 1978, Physical Processes in the Interstellar Medium (New York: Wiley)

Taffoni, G., Mayer, L., Colpi, M., \& Governato, F. 2003, MNRAS, 341, 434

Tozzi, P., Madau, P., Meiksin, A., \& Rees, M. J. 2000, ApJ, 528, 597

Tremaine, S., et al. 2002, ApJ, 574, 740

Tumlinson, J., \& Shull, J. M. 2000, ApJ, 528, L65

van den Bosch, F. C., Lewis, G. F., Lake, G., \& Stadel, J. 1999, ApJ, 515, 50

Venkatesan, A., Giroux, M. L., \& Shull, M. J. 2001, ApJ, 563, 1

Verner, D. A., Ferland, G. J., Korista, K. T., \& Yakovlev, D. G. 1996, ApJ, 465,487

Volonteri, M., Haardt, F., \& Madau, P. 2003a, ApJ, 582, 559 (Paper II)

Volonteri, M., Madau, P., \& Haardt, F. 2003b, ApJ, 593, 661 (Paper III)

Wasserburg, G. J., \& Qian, Y.-Z. 2000, ApJ, 538, L99

White, R. L., Becker, R. H., Fan, X., \& Strauss, M. A. 2003, AJ, 126, 1

Wiseman, A. 1992, Phys. Rev. D, 46, 1517

Wyithe, J. S. B., \& Loeb, A. 2003, ApJ, 588, L69

Yoshida, N., Abel, T., Hernquist, L., \& Sugiyama, N. 2003, ApJ, 592, 645

Yu, Q., \& Tremaine, S. 2002, MNRAS, 335, 965 